\title{
Las competencias transversales en los títulos de biblioteconomía y documentación: ¿avance o retroceso?
}

Generic skills in library and information science pregraduate programmes: progress or backward movement?

\section{Cristina faba-PÉrez, Francisco luis Rico Callado}

Departamento de Información y Comunicación, Facultad de Ciencias de la Documentación y la Comunicación, Universidad de Extremadura, \{cfabper|franciscorico@unex.es\}

\begin{abstract}
Resumen
El objetivo del presente trabajo es comparar la adquisición de competencias transversales implícitas en las antiguas titulaciones de Biblioteconomía y Documentación con la adquisición de competencias transversales explícitas del actual título de Grado en Información y Documentación que se imparte en la Universidad de Extremadura. Para ello, se trabaja con tres muestras: los alumnos de antiguos y nuevos estudios, los egresados de títulos antiguos y los docentes. Los resultados demuestran que no existe una diferencia significativa entre ambos estudios, lo que indica que dichas competencias se estaban impartiendo y adquiriendo de forma tácita en los planes antiguos.
\end{abstract}

Palabras clave: Competencias transversales. Biblioteconomía y Documentación. Universidad de Extremadura. Evaluación. Estudios de grado.

\section{Introducción: las competencias transversales en el marco del Espacio Europeo de Educación Superior (EEES)}

La bibliografía, tanto normativa como de carácter pedagógico o evaluativo sobre la implantación e implicaciones del Espacio Europeo de Educación Superior es ingente. En su base está la declaración de Bolonia, cuyo fin es desarrollar la educación superior, favoreciendo la comparabilidad y la compatibilidad de los estudios que se imparten en los países firmantes. Otro de sus objetivos, desarrollado en acuerdos posteriores es conectar la universidad con las exigencias planteadas por la sociedad del siglo XXI y, particularmente, el mundo laboral y empresarial. En este último ámbito, sin duda, las competencias se han convertido en el referente fundamental (Meneses Hernández, 2008-2009; Corominas, 2001).

En buena parte de la normativa tanto internacional como española no hay una definición del concepto de competencia. A partir de las diferentes aportaciones, se llega a la conclusión de que esta realidad se liga a la acción, esto es,

\begin{abstract}
The aim of this study is to compare the acquisition of generic skills implied in the former Library and Information Science (LIS) degrees with the acquisition of generic skills explicited in the current LIS studies at the University of Extremadura. Three samples were collected: students of both the former and new studies, graduates of the previous programmes, and teachers. The results show no significant difference between the two pregraduate programmes, thus indicating that generic skills were being tacitly imparted and acquired in the old studies.
\end{abstract}

Keywords: Generic skills. Library and information science. Pregraduate studies. University of Extremadura. Evaluation.

tiene un carácter práctico, relacionándose con una respuesta a una situación compleja y cambiante que puede ser desconocida por el individuo (Alonso Martín, 2010; Rué Domingo, 2008). Por otro lado, tiene dimensiones tanto cognitivas como no cognitivas, de modo que implica realidades como saber, saber hacer (procedimientos o habilidades) y saber ser (actitudes). Esto plantea, sin duda, un reto importante a los profesores universitarios, puesto que, si bien los conocimientos siguen siendo importantes, no son suficientes. Es más, estos adquieren significado en determinadas situaciones $y$, por tanto, tienen un carácter instrumental (Bajo, Maldonado, Moreno, 2003; Perrenoud, 1999). Hay otro elemento importante de la competencia que no podemos pasar por alto y que es la "idoneidad", esto es, se trata de una conducta observable que se puede evaluar a través de unos criterios claros (Tejada Artigas, 2006, p. 15-22; Rué Domingo, 2007).

Cabe decir que, aunque diseñado en un primer momento en Estados Unidos de América y Gran Bretaña en los años sesenta y setenta, el inte- 
rés por las competencias ha afectado de lleno a los países europeos y latinoamericanos. Respecto a su desarrollo tuvo una particular importancia el Proyecto Tuning (Montero Curiel, 2010) que brindó una clasificación que constituye el marco de referencia de las reformas. En este sentido, hay que destacar que se distinguen dos tipos esenciales: las transversales o genéricas y las específicas. A su vez, estas se dividen en tres grupos: instrumentales, interpersonales y sistémicas.

Las competencias profesionales, evidentemente, se relacionan con el desempeño laboral. Por esta razón, el diseño del currículo de la titulación requiere de un buen conocimiento del perfil laboral. En cuanto a las transversales, cabe decir, en principio, que inciden en la formación integral del individuo, cuestión en la que las universidades deben jugar un papel esencial de acuerdo con las directrices europeas (Corominas Rovira, 2011; Sánchez Elvira, López González, Fernández Sánchez, 2010); esto es lo que deja entrever el documento "Key Competences for Lifelong learning, european reference framework" (2004) y los programas "Education and Training" (2010), renovados con otro del mismo nombre y cuya fecha final está fijada en 2020.

De este modo, el paso de una universidad centrada en la enseñanza a otra estructurada sobre el aprendizaje implica que las competencias genéricas, en cuya evaluación se centra este trabajo, sean especialmente valoradas. Esto se debe a que son multifuncionales, es decir, se aplican a numerosas ocupaciones y situaciones, y motivan el desarrollo tanto personal como profesional. Constituyen, a su vez, un elemento común de las titulaciones (Alonso Martín, 2010). No hay que olvidar, sin embargo que, desde la psicología, especialmente entre los cognitivistas, se plantean serios obstáculos a las competencias transversales, circunstancia que se explica por las limitaciones de los individuos para aplicar una estructuras o habilidades a contenidos o situaciones diferentes a los que se experimentan en el curso de su aprendizaje (Rey, 2000).

Por otro lado, la inserción de las competencias en el engranaje de la educación superior para convertirlas en su eje central se relaciona con la flexibilidad de los programas de estudios, que deben de estar en consonancia con las necesidades de la sociedad y del sistema productivo. Esta concordancia entre ambos mercados, el educativo y el laboral, implica que el primero debe servir al segundo. Y esto es así, postulan sus partidarios, por razones de progreso social y económico. En efecto, estos sostienen que las reformas implican una mejora de las condiciones de vida y la formación de la población, mejorando el capital humano, que constituye, de acuerdo con su visión, el principal eje del desarrollo de un país (Beneitone, Esqueniti, González, Maletá, Suifi, y Wagenaar, 2007; Amaya, 2006). No es de extrañar, pues, que algunos autores hablen de "competencias productivas".

En diversas aportaciones, especialmente referidas a Latinoamérica, se resalta que esta sumisión de la formación a las exigencias del mercado laboral entraña la imposición de un modelo neoliberal en el ámbito académico. En consecuencia, en la universidad moderna, en el proceso educativo deben primar las habilidades que elevan la cualificación de los trabajadores en función de las exigencias de las empresas. De este modo, se hace hincapié en la idea de que la universidad debe dar una salida a sus titulados, ajustando su oferta a las posibilidades laborales. Tal idea ha calado en la sociedad y la evaluación del aprendizaje de las competencias a través de las encuestas del alumnado de los últimos cursos de las licenciaturas o de los egresados es reveladora de esto (García Manjón y Pérez López, 2008; Ornelas, 2007; Fuentes Navarro, 2007; Posada Álvarez, 2008).

Cabe decir, por otro lado, que la imagen que se da, en una buena parte de la bibliografía, de los estudios universitarios anteriores oponiéndolos a la organización propuesta por el EEES, aunque acertada en algunos aspectos, resulta simplista en tanto que los presenta como antediluvianos desde el punto de vista de la metodología, la evaluación e, implícitamente, respecto a su capacidad para formar profesionales en la sociedad de la información.

Esta defensa a ultranza y acrítica, asentada en la opinión pública y las instituciones políticas y académicas, del nuevo modelo sin considerar y discutir sus fundamentos y efectos resulta, cuando menos, injusta respecto a la enseñanza universitaria vigente hasta el momento (Barnett, 2001). En efecto, de alguna manera las competencias estaban ya insertas en las antiguas licenciaturas y diplomaturas. El adiestramiento en ellas no era ni mucho menos desconocido para los docentes, si bien faltaban una conciencia y una planificación apoyadas en una metodología docente y un sistema de evaluación adecuado Así por ejemplo, se cita en algunos trabajos la expresión oral, la escrita, el trabajo autónomo o la resolución de problemas (Delgado García, 2005, p. 24).

Por esta razón, una propuesta que tenga en cuenta tanto a los docentes como a los alumnos de las antiguas titulaciones (estudiantes y egre- 
sados) para comparar sus opiniones sobre la adquisición de competencias genéricas con las de los grados actuales, no resulta gratuita. EI objetivo del presente trabajo es comparar la adquisición de competencias transversales implícitas en las antiguas titulaciones de Biblioteconomía y Documentación con la adquisición de competencias transversales explícitas del actual Título de Grado en Información y Documentación (INDO) que se imparte actualmente en la Universidad de Extremadura (Unex). La finalidad es comprobar si existe una diferencia significativa entre ambos estudios a favor de los nuevos grados o, por el contrario, dichas competencias se estaban impartiendo y adquiriendo en los planes antiguos.

\section{Materiales y metodología}

En este trabajo se presentan algunos de los resultados relacionados con las competencias transversales del Proyecto de Innovación Docente titulado "Proyecto para la Mejora de la Calidad Docente" (A), desarrollado durante el curso académico 2009-2010 y del Grupo de Innovación Didáctica titulado "¿Qué ha ocurrido con la motivación del profesor y su implicación en un proyecto universitario común?" (B), realizado en el curso 2010-2011 en la Facultad de Ciencias de la Documentación y la Comunicación de la Universidad de Extremadura, concedidos ambos por el Vicerrectorado de Calidad de la Universidad de Extremadura y dirigidos por Cristina Faba Pérez.

En el primer Proyecto (A) se trabajó con dos muestras que fueron seleccionadas entre los:

- Estudiantes y egresados de los títulos antiguos Diplomatura en Biblioteconomía y Documentación y Licenciatura en Documentación de la Unex que respondieron a un cuestionario que fue diseñado y enviado previamente on line a la población total de estudiantes y egresados suministrada por el Centro que ascendía a 2338. De ellos, respondieron 50 que constituyen la muestra que empleamos.

- Docentes de dichas titulaciones que respondieron al cuestionario enviado. Del total de la población formada por 33 profesores respondieron 20 que conforman la muestra.

En el segundo Proyecto (B) se trabajó con una muestra seleccionada del siguiente modo:

- Alumnos de las asignaturas de $1^{\circ}$ de Grado en Información y Documentación que cumplimentaron el cuestionario repartido en las aulas. Las asignaturas fueron seleccionadas a partir de los siguientes criterios: que se es- tuvieran impartiendo en el semestre de concesión del proyecto y que los profesores implicados en las mismas formaran parte del grupo de innovación didáctica del proyecto, independientemente del área de conocimiento puesto que se trataba de analizar el título. los alumnos que cumplimentaron el cuestionario de cada asignatura fueron 15.

En ambos casos, la variable que se analizó en cada uno de los cuestionarios fue la lista de competencias transversales recogidas en el Título de Grado en INDO de la Unex, a las que añadieron cuatro nuevas (OC) que se consideraron imprescindibles en Información y Documentación. En la tabla I aparecen junto al código que se les ha asignado en el Título (Libro blanco..., 2004; Mano González, Moro Cabero, 2009).

\begin{tabular}{|c|c|}
\hline Cód. & Competencia \\
\hline C18 & $\begin{array}{l}\text { Capacidad de análisis y síntesis aplicadas a la } \\
\text { gestión y organización de la información }\end{array}$ \\
\hline C19 & $\begin{array}{l}\text { Capacidad de uso y adaptación de diversas técnicas } \\
\text { de comunicación oral y escrita con los usuarios de la } \\
\text { información }\end{array}$ \\
\hline $\mathrm{C} 20$ & $\begin{array}{l}\text { Habilidades en el uso de software genérico } \\
\text { (ofimática) }\end{array}$ \\
\hline $\mathrm{C} 21$ & $\begin{array}{l}\text { Buen conocimiento hablado y escrito de una lengua } \\
\text { extranjera }\end{array}$ \\
\hline $\mathrm{C} 22$ & $\begin{array}{l}\text { Capacidad de organización y planificación del } \\
\text { trabajo propio }\end{array}$ \\
\hline $\mathrm{C} 23$ & $\begin{array}{l}\text { Capacidad de trabajar en equipo y de integración en } \\
\text { equipos multidisciplinares }\end{array}$ \\
\hline $\mathrm{C} 24$ & $\begin{array}{l}\text { Reconocimiento de la diversidad y la } \\
\text { multiculturalidad en el trabajo en el servicio público }\end{array}$ \\
\hline $\mathrm{C} 25$ & $\begin{array}{l}\text { Razonamiento crítico en el análisis y la valoración de } \\
\text { alternativas }\end{array}$ \\
\hline $\mathrm{C} 26$ & $\begin{array}{l}\text { Compromiso ético en las relaciones con los usuarios } \\
\text { y en la gestión de la información }\end{array}$ \\
\hline $\mathrm{C} 27$ & Capacidad para el aprendizaje autónomo \\
\hline C28 & $\begin{array}{l}\text { Capacidad para la adaptación a cambios en el } \\
\text { entorno }\end{array}$ \\
\hline $\mathrm{C} 29$ & $\begin{array}{l}\text { Capacidad para emprender mejoras y proponer } \\
\text { innovaciones }\end{array}$ \\
\hline $\mathrm{C} 30$ & $\begin{array}{l}\text { Capacidad de dirección, liderazgo y toma de } \\
\text { decisiones }\end{array}$ \\
\hline C31 & Capacidad de generar una conciencia solidaria \\
\hline OC1 & $\begin{array}{l}\text { Uso de Internet como medio de comunicación y } \\
\text { fuente de información }\end{array}$ \\
\hline OC2 & Resolución de problemas \\
\hline OC3 & Capacidad para trabajar en un contexto internacional \\
\hline OC4 & Motivación por suministrar un servicio de calidad \\
\hline
\end{tabular}

Tabla I. Competencias transversales del Título de Grado en INDO de la Unex 
En el Proyecto A se diseñó un cuestionario donde se valoraba de 1 a 5 -siendo 1 la nota mínima y 5 la máxima- la adquisición de las competencias transversales durante la titulación, en el caso de los estudiantes y egresados, y la enseñanza tácita de las mismas por parte de los docentes. Es decir, se trataba de detectar, entre otras cosas, si los alumnos y profesores las habían recibido o impartido aún sin estar regladas.

En el Proyecto B la muestra de estudiantes valoró también del 1 al 5 el cumplimiento de las competencias transversales en los programas docentes de sus asignaturas del Grado en INDO. A este respecto, se trató de descubrir, entre otras cosas, si habían recibido en sus clases las que aparecían en los programas.

\section{Resultados}

\subsection{Proyecto $A$}

En la tabla II se muestran las valoraciones de los estudiantes y egresados de los títulos antiguos. En términos generales se puede considerar que las competencias transversales obtuvieron un aprobado, es decir, que se habían adquirido, puesto que la nota media fue de 3,27 sobre 5. Curiosamente, las dos más valoradas no figuran en el plan de estudios actual del Grado de INDO. Por tanto, los participantes compartían la sensación de haber recibido una buena formación en el ámbito de unas competencias que "oficialmente" no se consideran imprescindibles en el Título.

\begin{tabular}{llr}
\hline Cód. & Competencias & Prom. \\
\hline OC1 & Uso de Internet como medio de comunicación y fuente de información. & 4,00 \\
\hline OC4 & Motivación por suministrar un servicio de calidad al usuario. & 3,90 \\
\hline C23 & Capacidad de trabajar en equipo y de integración en equipos multidisciplinares. & 3,71 \\
\hline C22 & Capacidad de organización y planificación del trabajo propio. & 3,71 \\
\hline C26 & Compromiso ético en las relaciones con los usuarios y en la gestión de la información. & 3,71 \\
\hline C27 & Capacidad para el aprendizaje autónomo & 3,65 \\
\hline C18 & Capacidad de análisis y síntesis para la gestión y la organización de la información & 3,57 \\
\hline C20 & Habilidades en el uso de software genérico (ofimática) & 3,55 \\
\hline C28 & Capacidad para la adaptación a cambios en el entorno & 3,45 \\
\hline C19 & Capacidad de uso y adaptación de técnicas de comunicación oral y escrita con los usuarios de la información & 3,31 \\
\hline C30 & Capacidad de dirección, liderazgo y toma de decisiones & 3,08 \\
\hline OC2 & Resolución de problemas y estudio de casos & 3,08 \\
\hline C24 & Reconocimiento de la diversidad y la multiculturalidad en el trabajo en el servicio público & 3,06 \\
\hline C31 & Capacidad de generar una conciencia solidaria & 3,06 \\
\hline C25 & Razonamiento crítico en el análisis y la valoración de alternativas. & 3,00 \\
\hline C29 & Capacidad para emprender mejoras y proponer innovaciones & 2,96 \\
\hline OC3 & Capacidad para trabajar en un contexto internacional & 2,33 \\
\hline C21 & Conocimiento hablado y escrito de una lengua extranjera & 1,76 \\
\hline Nota media de las competencias transversales & 3,27 \\
\hline & &
\end{tabular}

Tabla II. Estudiantes y egresados: valoración de la transversalidad en los títulos antiguos

Como puede verse en el extremo inferior de la tabla II, los últimos valores corresponden a dos capacidades que, dada la evolución de la sociedad y de las empresas, son cada vez más necesarias.

En la tabla III se muestran las calificaciones asignadas por los docentes. Al igual que ocurría con los estudiantes, obtuvieron un aprobado, con una media de 3,68 sobre 5 . Las mejor calificadas fueron aquellas que, nuevamente, no se han considerado en el plan de INDO de la Universidad de Extremadura. Como en el caso de los alumnos, el "conocimiento de la lengua extranjera" se sitúa al final de la distribución. 


\begin{tabular}{|c|c|c|}
\hline Cód. & Competencias & Promedio \\
\hline OC1 & Uso de Internet como medio de comunicación y fuente de información & 4,21 \\
\hline OC4 & Motivación por suministrar un servicio de calidad & 4,16 \\
\hline $\mathrm{C} 27$ & Capacidad para el aprendizaje autónomo & 4,11 \\
\hline C18 & Capacidad de análisis y síntesis para la gestión y organización de la información & 4,05 \\
\hline OC2 & Resolución de problemas y estudio de casos & 4,05 \\
\hline $\mathrm{C} 20$ & Habilidades en el uso de software genérico (ofimática) & 4,00 \\
\hline $\mathrm{C} 25$ & Razonamiento crítico en el análisis y la valoración de alternativas & 3,89 \\
\hline $\mathrm{C} 28$ & Capacidad para la adaptación a cambios en el entorno & 3,84 \\
\hline $\mathrm{C} 22$ & Capacidad de organización y planificación del trabajo propio & 3,79 \\
\hline C19 & Capacidad de uso y adaptación de diversas tcas de c. oral y escrita con los usuarios de la información & 3,79 \\
\hline $\mathrm{C} 26$ & Compromiso ético en las relaciones con los usuarios y en la gestión de la información & 3,74 \\
\hline $\mathrm{C} 23$ & Capacidad de trabajar en equipo y de integración en equipos multidisciplinares & 3,47 \\
\hline C29 & Capacidad para emprender mejoras y proponer innovaciones & 3,47 \\
\hline C30 & Capacidad de dirección, liderazgo y toma de decisiones & 3,42 \\
\hline OC3 & Capacidad para trabajar en un contexto internacional & 3,32 \\
\hline $\mathrm{C} 24$ & Reconocimiento de la diversidad y la multiculturalidad en el trabajo en el servicio público & 3,26 \\
\hline C31 & Capacidad de generar una conciencia solidaria & 3,11 \\
\hline $\mathrm{C} 21$ & Conocimiento hablado y escrito de una lengua extranjera & 2,47 \\
\hline \multicolumn{2}{|c|}{ Nota media de las competencias transversales } & 3,68 \\
\hline
\end{tabular}

Tabla III. Docentes: valoración de la transversalidad en los títulos antiguos

En el gráfico 1 se puede observar que la correlación Pearson entre la distribución de competencias por parte de ambas muestras es muy fuerte ya que asciende a $r=0,80$. (Según Ferreiro-Aláez (1993) es muy fuerte si es superior a 0,700 ; sustancial, entre 0,500 y 0,699 ; y moderada entre 0,300 y 0,499.) Además, coinciden los valores más altos en la adquisición e impartición de la transversalidad (OC1: uso de Internet como medio de comunicación y fuente de información; OC4: motivación por suministrar un servicio de calidad) y el valor más bajo de la distribución (C21: conocimiento hablado y escrito de una lengua extranjera).

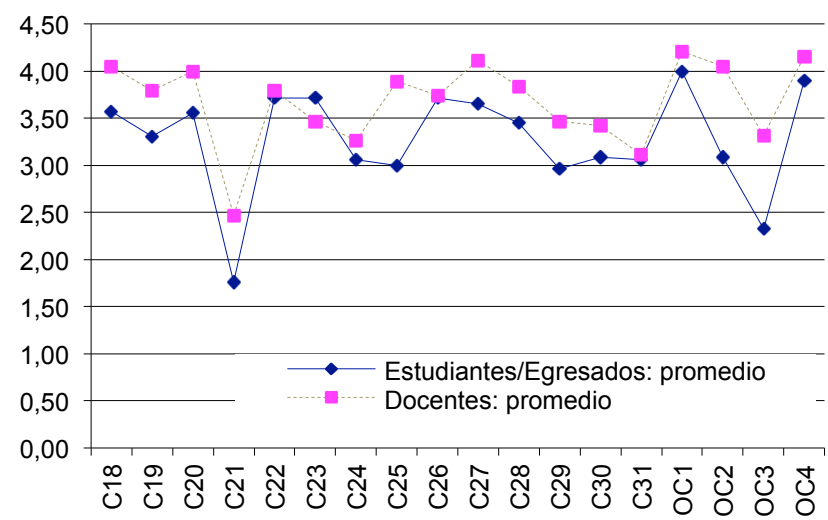

Gráfíco 1. Relación entre las competencias transversales en los estudiantes y egresados y los Docentes.

\subsection{Proyecto $B$}

En este caso, se trataba de comprobar si en las asignaturas de $1^{\circ}$ de Grado en INDO se estaban impartiendo las competencias transversales. Para ello, se diseñó un cuestionario que recogía tres tipos de competencias: a) las contenidas en el título oficial y citadas por el profesor en el programa de la asignatura. Por tanto, se podían considerar de obligado cumplimiento por su parte (en cursiva); b) otras contenidas en el título aunque no citadas expresamente por el profesor en el programa, si bien se podían estar transmitiendo sin tener conciencia de ello ( $\sin$ destaque); c) las detectadas por los alumnos y que no figuran en el actual título oficial (OC) (en negrita). Las asignaturas analizadas fueron "Diplomática y producción documental en las instituciones" e "Introducción a la estadística documental".

En la tabla IV se muestran los resultados obtenidos por "Diplomática". Se puede observar que la nota media es de 2,41 sobre 5 . Es positivo que todas las competencias recogidas en el programa docente, en cursiva, se sitúen entre los valores más elevados de la distribución. Al igual que ocurrió en el Proyecto A, una ausente del título oficial, en negrita, fue la mejor valorada de todas $(3,08$ OC4 "Motivación por suministrar un servicio de calidad"). 


\begin{tabular}{llc}
\hline Cód. & Competencia & Promedio \\
\hline OC4 & Motivación por suministrar un servicio de calidad & $\mathbf{3 , 0 8}$ \\
\hline C22 & Capacidad de organización y planificación del trabajo propio. & 2,87 \\
\hline C25 & Razonamiento crítico en el análisis y la valoración de alternativas & 2,79 \\
\hline C27 & Capacidad para el aprendizaje autónomo. & 2,79 \\
\hline C18 & Capacidad de análisis y síntesis aplicadas a la gestión y organización de la información & 2,67 \\
\hline C19 & Capacidad de uso y adaptación de diversas tcas de c. oral y escrita con los usuarios de la información & 2,64 \\
\hline C26 & Compromiso ético en las relaciones con los usuarios y en la gestión de la información & 2,64 \\
\hline OC2 & Resolución de problemas & 2,58 \\
\hline C30 & Capacidad de dirección, liderazgo y toma de decisiones. & 2,57 \\
\hline C29 & Capacidad para emprender mejoras y proponer innovaciones & 2,54 \\
\hline C31 & Tener la capacidad de generar una conciencia solidaria & 2,54 \\
\hline C24 & Reconocimiento de la diversidad y la multiculturalidad en el trabajo en el servicio público & 2,38 \\
\hline OC3 & Capacidad para trabajar en un contexto internacional & 2,33 \\
\hline C28 & Capacidad para la adaptación a cambios en el entorno & 2,31 \\
\hline C23 & Capacidad de trabajar en equipo y de integración en equipos multidisciplinares & 1,83 \\
\hline C20 & Habilidades en el uso de software genérico (ofimática) & 1,77 \\
\hline OC1 & Uso de Internet como medio de comunicación y fuente de información & $\mathbf{1 , 6 2}$ \\
\hline C21 & Buen conocimiento hablado y escrito de una lengua extranjera & 1,50 \\
\hline Nota media de las competencias transversales & 2,41 \\
\hline Tabla & C. Asianatura: Diplomática y producción documental en las instituciones. comptencias transversales.
\end{tabular}

Tabla IV. Asignatura: Diplomática y producción documental en las instituciones, competencias transversales.

\begin{tabular}{llc}
\hline Cód. & Competencia & Promedio \\
\hline C18 & Capacidad de análisis y síntesis aplicadas a la gestión y organización de la información. & 3,36 \\
\hline C22 & Capacidad de organización y planificación del trabajo propio & 3,29 \\
\hline OC2 & Resolución de problemas & $\mathbf{3 , 2 0}$ \\
\hline OC4 & Motivación por suministrar un servicio de calidad & $\mathbf{3 , 0 8}$ \\
\hline C25 & Razonamiento crítico en el análisis y la valoración de alternativas & 3,07 \\
\hline C27 & Capacidad para el aprendizaje autónomo & 3,07 \\
\hline C20 & Habilidades en el uso de software genérico (ofimática) & 2,86 \\
\hline C28 & Capacidad para la adaptación a cambios en el entorno & 2,85 \\
\hline C19 & Capacidad de uso y adaptación de diversas tcas de c. oral y escrita con los usuarios de la información & 2,77 \\
\hline C31 & Capacidad de generar una conciencia solidaria & 2,77 \\
\hline C30 & Capacidad de dirección, liderazgo y toma de decisiones & 2,75 \\
\hline C26 & Compromiso ético en las relaciones con los usuarios y en la gestión de la información & 2,54 \\
\hline C29 & Capacidad para emprender mejoras y proponer innovaciones & 2,46 \\
\hline OC3 & Capacidad para trabajar en un contexto internacional & $\mathbf{2 , 4 6}$ \\
\hline C24 & Reconocimiento de la diversidad y la multiculturalidad en el trabajo en el servicio público & 2,38 \\
\hline OC1 & Uso de Internet como medio de comunicación y fuente de información & 2,36 \\
\hline C23 & Capacidad de trabajar en equipo y de integración en equipos multidisciplinares & $\mathbf{2 , 1 5}$ \\
\hline C21 & Buen conocimiento hablado y escrito de una lengua extranjera & 1,23 \\
\hline Nota media de las competencias transversales & 2,70 \\
\hline & & 23 \\
\hline
\end{tabular}

Tabla V. Asignatura: Introducción a la estadística documental, competencias transversales. 
En la tabla $V$ figuran los datos correspondientes a "Introducción a la estadística". La media es de 2,70 sobre 5 . Nuevamente, las recogidas en el programa obtuvieron las puntuaciones más elevadas. Se produjo, finalmente, otra coincidencia con los casos reseñados anteriormente, puesto que dos de las que no se contemplan oficialmente, en negrita, aparecieron bien situadas (OC2 y OC4).

En el gráfico 2 se muestra una relación muy fuerte entre ambas asignaturas $(r=0,74)$. De nuevo, la competencia peor valorada en ambas materias coincide con el Proyecto A (C21: conocimiento hablado y escrito de una lengua extranjera). En este caso, la segunda mejor posicionada en las dos asignaturas corresponde a la transversal C22: Capacidad de organización y planificación del trabajo propio.

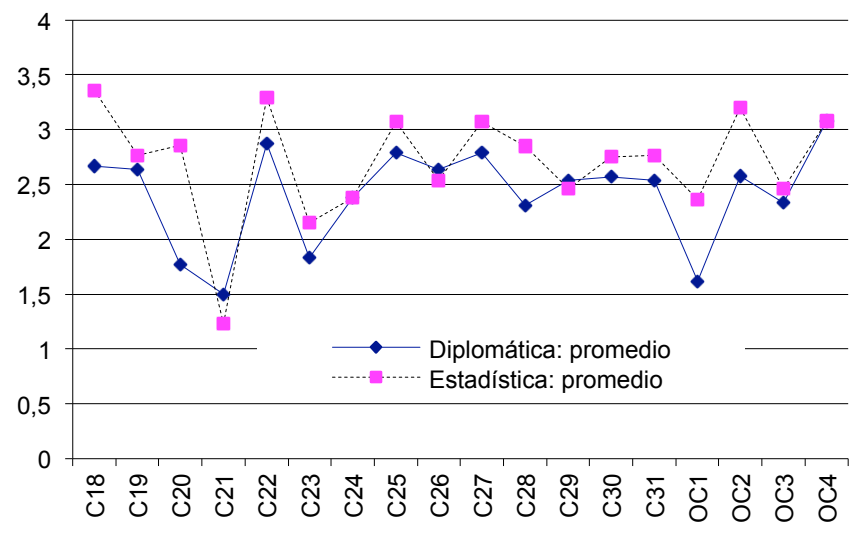

Gráfico 2. Las competencias transversales en el proyecto $B$

\section{Conclusiones}

Las principales impresiones que se derivan de este trabajo son las siguientes.

En términos generales, se puede considerar que en los títulos antiguos de Biblioteconomía y Documentación de la Unex se impartían y recibían competencias transversales, aunque las mismas no se contemplasen en las titulaciones oficiales. Es más, obtenían una nota respetable ( 3,25 y 3,68 sobre 5 en el colectivo de estudiantes y egresados y de docente, respectivamente). Sin embargo, debido a la pequeña muestra de estudiantes y egresados que respondieron al cuestionario enviado, es arriesgado extrapolar dichos resultados más allá de esta investigación, lo que nos motiva a seguir trabajando en este campo.

En los nuevos estudios de Grado, la muestra observada nos indica una apreciación menor en cuanto a la impartición y enseñanza de las mismas, a pesar de que, paradójicamente, son un elemento sustancial de los programas. Al igual que en el caso anterior, es preciso seguir trabajando en este ámbito ampliando el número de asignaturas del título a evaluar.

En ambos proyectos, se valoraron positivamente una serie de competencias no contempladas en el título oficial, tales como: el "uso de Internet como medio de comunicación y fuente de información" y la "motivación por suministrar un servicio de calidad". Esto significa que se impartieron de forma "intuitiva" dos habilidades esenciales en la actualidad para el desarrollo tanto profesional como personal en el ámbito de la Información y la Documentación. Por el contrario, obtuvieron un índice muy deficiente otras como un "buen conocimiento hablado y escrito de una lengua extranjera", que sigue siendo una asignatura pendiente de la universidad (Pulgar, 2009) y la "capacidad para trabajar en un contexto internacional".

Tales datos dejan entrever que el objetivo del profesorado sigue siendo, fundamentalmente, la adquisición de conocimientos o habilidades destinadas al desarrollo profesional. De este modo, el tiempo y el esfuerzo dedicados al desarrollo de las competencias transversales que se relacionan más directamente con esto es notable, circunstancia que se debe, evidentemente, a que el profesor las identifica e incluye en sus programas. Sin embargo, aquellas que se alejan de esta meta son minusvaloradas. Cabe decir, pues, que las competencias siguen siendo asumidas de un modo instrumental, con el fin de impartir la asignatura concreta, circunstancia que se documenta en otros trabajos (Rosich y Bosch, 2008). La valoración que el profesorado universitario hace de las competencias en su actividad docente es un aspecto que, sin duda, requiere un estudio más profundo (Cáceres Lorenzo y Salas Pascual, 2012).

La conclusión no puede ser otra, y en esto coincidimos con otros autores (Alonso Martín, Pilar, 2010; Mir Acebrón, 2008), que resaltar la importancia de realizar un trabajo común entre las diferentes asignaturas que componen los grados. Necesariamente, estas deben complementarse para desarrollar óptimamente las competencias genéricas haciéndolas ajenas de un "contenido" determinado. Sólo así, en principio, se pueden adquirir de un modo pleno y favorecer la adaptabilidad del alumnado a unas condiciones sociales y laborales cambiantes. No en vano, el pleno desarrollo del EEES implica una ruptura de la "estanqueidad" de la asignatura tradicional. Esto se puede conseguir mediante los seminarios y las tutorías (San Antonio Gó- 
mez y Manzano Agugliaro, 2010; Pérez Cañado, 2011); o bien mediante trabajos que relacionen varias asignaturas de un grado (Todolí Torrí et alii, 2009; Rodríguez Esteban, Ausín Zorrilla, Revilla, 2007; Ion y Cano, 2011).

Finalmente, una cuestión que es preciso destacar se refiere al grado de conocimiento que los alumnos tienen de las competencias, su significado y el modo de adquirirlas a través del desarrollo de las asignaturas. Sin minusvalorar la experiencia de la secundaria, claro ejemplo del concepto de competencia que se ha extendido a otras etapas de la enseñanza, hemos de plantearnos en qué medida la información de los programas docentes e, incluso, de estos trabajos de evaluación resulta suficiente para el usuario y si sus resultados no son, a menudo, sesgados. A este respecto, y con la perspectiva de mejorar futuras aportaciones debe considerarse, necesariamente, la posibilidad de introducir las subcompetencias tanto en el de diseño de los programas como de las asignaturas como en las encuestas (Baartman y Ruijs, 2011).

\section{Agradecimientos}

Este trabajo ha sido financiado por la Junta de Extremadura, Consejería de Empleo, Empresa e Innovación y el Fondo Social Europeo como parte de la ayuda a grupos GR10019.

\section{Referencias}

Alonso Martín, Pilar (2010). La valoración de la importancia de las competencias transversales: comparación de su percepción al inicio y final de curso en alumnos de Psicología. // Revista de investigación educativa. 28:1 (2010) 119-140.

Amaya, L. (2006). La educación basada en competencias: implicaciones, retos y perspectivas. // Didac. 14 (2006) 26-33.

Baartman, Liesbeth; Ruijs, Lotte (2011). Comparing students perceived and actual competence in higher vocational education. // Assessment and Evaluation in Higher Education. 36:4 (2011) 385-398.

Bajo, $\mathrm{M}^{\mathrm{a}}$ Teresa; Maldonado, Antonio; Moreno, Sergio; Moya, Miguel; Tudel, Pío (2003). Las competencias en el nuevo paradigma educativo para Europa. Granada. Universidad de Granada, 2003.

Barnett, Ronald (2001). Los límites de la competencia: EI conocimiento, la educación superior y la sociedad. Barcelona: Gedisa, 2001.

Beneitone, P.; Esqueniti, C.; González, J.; Maletá, M.; Suifi, G.; Wagenaar, R. (2007). Reflexiones y perspectivas de la Educación Superior en América Latina: Informe FinalProyecto Tuning-América Latina 2004-2007. Bilbao: Universidad de Deusto, 2007. http://tuning-unide usto.org/tunignal/index.php?option=com_docman\&task= own\&bid=54 (2011-04-22)

Cáceres Lorenzo, María Teresa; Salas Pascual, Marcos (2012). Valoración del profesorado sobre las competencias genéricas: su efecto en la docencia. // Revista lberoamericana de Psicología y Salud. 3:2 (2012) 195-210. http://www.usc.es/suips/Revista/caceres.pdf (20-062012).
Corominas Rovira, Enric (2001). Competencias genéricas en la formación universitaria. // Revista de educación. 325 (2001) 200-321.

Delgado García, Ana M. (coord.) (2005). Competencias y diseño de la evaluación continua y final en el Espacio europeo de educación superior. Madrid: Ministerio de Educación y Ciencia, 2005. Programa de estudios y análisis. http://nevada.ual.es:81/ufid/archivos/competen cias\%20evaluacion\%20ees-MEC.pdf (2011-04-11).

Ferreiro Aláez, L. (1993). Bibliometría: análisis bivariante. Madrid: Espasa, 1993.

Fuentes Navarro, M. T. (2007). Las competencias académicas desde la perspectiva interconductual. // Acta colombiana de Psicología. 10:2 (2007) 51-58.

García Manjón, J. V.; Pérez López, M. C. (2008). Espacio europeo de educación superior, competencias profesionales y empleabilidad. // Revista iberoamericana de educación superior. 46:9 (2008) 1-12.

Ion, Georgeta; Cano, Elena (2011). Asessment practices at Spanish universities: from a learning to a competence approach. // Evaluation and Research in Education. 24:3 (2011) 167-181.

Libro blanco de grado en Información y Documentación (2004). Madrid: Agencia Nacional de Evaluación de la Calidad y Acreditación. http://www.aneca.es/media/1504 24/libroblanco_jun05_documentacion.pdf\#10 (18/05/2012)

Mano González, Marta; Moro Cabero, Manuela (2009). La evaluación por competencias: propueta de un sistema de medida para el grado de Información y Documentación. // BID: Textos Universitaris de Biblioteconomia i documentació. 23 (2009). http://www.ub.edu/bid/23/de lamano2.htm (18-05-2012).

Meneses Hernández, Jorge A. (2008-2009). Competencias del profesional graduado de la Escuela de Bibliotecología, documentación e información de la Universidad Nacional de Costa Rica en la sociedad de la información. // Ístmica. 12 (2008-2009) 81-96.

Mir Acebrón, Anna (2008). Las competencias transversales en la Universidad Pompeu Fabra: La visión de los docentes y estudiantes de segundo ciclo. // REDU: Revista de docencia universitaria. 1 (2008) 1-16.

Montero Curiel, M (2010). El proceso de Bolonia y las nuevas competencias. // Tejuelo. 9 (2010) 19-37.

Ornelas, C. (2007). Educación y neoliberalismo en México. BUAP-Secretaría de Cultura del Estado de Puebla: México, 2007.

Pérez Cañado, María L. (2011). El desarrollo de competencias comunicativas a través de seminarios transversales ECTS: una experiencia en la Universidad de Jaén. // TESI: Teoría de la educación, educación y cultura en la sociedad de la información. 12:1 (2011) 294-319.

Perrenoud, P. (1999). Construir competencias desde la escuela. Santiago de Chile: Dolmen, 1999.

Posada Álvarez, R. (2008). Formación superior basada en competencias, interdisciplinariedad y trabajo autónomo del estudiante. // Revista iberoamericana de Educación. 19 (2008). http://www.rieoei.org/deloslectores/648Posa da.PDF (2011-03-17).

Pulgar, L. (coord.) (2009). Encuesta piloto sobre competencias genéricas a egresados de la UNED. Vicerrectorado de Innovación y apoyo docente. Instituto Universitario de Educación a Distancia (UNED). http://portal.uned.es/pls/ portal/docs/PAGE/UNED MAIN/LAUNIVERSIDAD/VICE RRECTORADOS/CALIDĀD E INNOVACION/INNOVA CION_DOCENTE/IUED/INSTITUCIONAL/INFORMECO MPETENCIASGEN\%C3\%89RICAS_080909.PDF (2011-05-03) 
Rodríguez Esteban, M. A.; Ausín Zorrilla, T.; Revilla, I, Vivar Quintana, A. M.; Martín Izard, J. F. (2007). Transversalidad de disciplinas en la titulación de Arquitectura Técnica. // El espacio europeo de educación, una oportunidad para las enseñanzas técnicas: Zamora 19, 20, 21y 22 de junio de 2007. Actas del congreso. Salamanca: Universidad de Salamanca, 2007.

Rey, B. (2000): ¿Existen las competencias transversales?. // Educar. 26 (2000) 9-17.

Rosich, L.; Bosch, F. (2008). Del escritorio a la tarima: una experiencia de formación en competencias transversales dentro de la licenciatura en Biología. // Educación médica. 11:1 (2008) 37-41.

Rué Domingo, J (2008). Formar en competencias en la universidad: entre la relevancia y la banalidad. // Red U. Revista de Docencia. Número monográfico 1: Formación centrada en competencias (2008). http://www.redu.m.es/ Red_U/m1. (2010-07-22).

Rué Domingo, J. (2007). Enseñar en la universidad: el EEES como reto para la educación superior. Madrid: Narcea, 2007.

San Antonio-Gómez, C.; Manzano-Agugliaro (2010). La tutoría como vehículo de fomento de las competencias en los nuevos grados de ingeniería agronómica. // Espiral: Cuadernos de profesorado. 3:6 (2010) 74-83.

Sánchez-Elvira, Ángeles; López-González, M., FernándezSánchez, M. (2010). Análisis de las competencias genéricas en los nuevos títulos de grado del EEES en las universidades españolas. // Revista de docencia universitaria. 8:1 (2010) 35-73

Tejada Artigas, Carlos: et alii (2006). El diseño del plan docente en Información y Documentación acorde con el Espacio Europeo de Educación Superior: un enfoque por competencias. Madrid: Universidad Complutense, 2006. $15-22$.

Todolí Torrí, J. L.; et alii (2009). Trabajo interdisciplinar como herramienta para fomentar el desarrollo de competencias transversales. // Goméz Lucas, C. y Grau Company, S. (eds). Propuestas de diseño, desarrollo e innovaciones curriculares y metodología en el EEES. Barcelona: Marfil, 2009. 701-720.

Enviado: 2012-05-14. Versión corregida: 2012-07-12. Aceptado: 2012-08-20. 
